\title{
Cisplatin-loaded hollow gold nanoparticles for laser-triggered release
}

\author{
Chiyi Xiong ${ }^{1}$, Wei Lu ${ }^{1,2}$, Min Zhou ${ }^{1,3}$, Xiaoxia Wen ${ }^{1}$ and Chun Li ${ }^{1 *}$ (B)
}

*Correspondence:

cli@mdanderson.org

${ }^{1}$ Department of Cancer

Systems Imaging, The

University of Texas MD

Anderson Cancer Center,

Houston, USA

Full list of author information

is available at the end of the

article

\begin{abstract}
Background: Hollow gold nanoparticles (HGNPs) exposed to near-infrared (NIR) light yield photothermal effects that can trigger a variety of biological effects for potential biomedical applications. However, the mechanism of laser-triggered drug release has not been studied before.
\end{abstract}

Methods: A tripeptide Ac-Glu-Glu-Cys- $\mathrm{NH}_{2}$ (Ac-EEC) was directly linked to the surface of HGNPs. The EEC-HGNPs conjugate was then complexed with cisplatin Pt(II) to give Ac-EEC(Pt)-HGNPs. Folic acid was introduced to the gold surface of Ac-EEC-HGNPs through a thioctic acid-terminated polyethylene glycol linker (F-PEG-TA) followed by complexation with Pt(II) to give F-Ac-EEC(Pt)-HGNPs. Laser treatment was instituted with a 15-ns pulsed laser at a repetition rate of $10 \mathrm{~Hz}$. The released $\mathrm{Pt}(\mathrm{II})$ was quantified by inductively coupled plasma mass spectroscopy, and the nature of the released Ptcontaining species was characterized by liquid chromatography-mass spectroscopy. The cytotoxicity was studied using the MTT assay.

Results: Pt(II) was released from Ac-EEC(Pt)-HGNPs via two modes: (1) sustained release through an inverse ligand exchange reaction with chloride ions and (2) rapid release through cleavage of the $\mathrm{Au}-\mathrm{S}$ bond between the tripeptide linker and $\mathrm{Au}$ surface upon NIR laser irradiation. The folate (F) conjugate of the nanoconstruct, F-AcEEC(Pt)-HGNPs, in combination with laser treatment showed a significantly greater effect on cell mortality against folate-overexpressing human epidermoid carcinoma KB cells than F-Ac-ECC(Pt)-HGNPs alone after $24 \mathrm{~h}$ of incubation.

Conclusions: These results demonstrate that the photothermal property of HGNPS can be used for dual-modality photothermal therapy and NIR laser-triggered platinumbased chemotherapy.

Keywords: Cisplatin, Hollow gold nanoparticles, Near-infrared light, Controlled release, Photothermal conversion

\section{Background}

Among the inorganic nanoparticles, gold nanoparticles have garnered the greatest attention for biomedical applications. Gold nanoparticles exhibit a unique and tunable optical property, termed surface plasmon resonance (SPR), which accounts for their photothermal effects (Jain et al. 2007). Gold nanoparticles also exhibit excellent biocompatibility (Bhattacharya and Mukherjee 2008; Connor et al. 2005; You et al. 2014) and have been investigated as carriers for various anticancer agents (Cheng et al. 2008; Ghosh et al. 2008; Gibson et al. 2007; Kim et al. 2009; Lee et al. 2009).

(c) The Author(s) 2018. This article is distributed under the terms of the Creative Commons Attribution 4.0 International License (http://creativecommons.org/licenses/by/4.0/), which permits unrestricted use, distribution, and reproduction in any medium, provided you give appropriate credit to the original author(s) and the source, provide a link to the Creative Commons license, and indicate if changes were made. 
Hollow gold nanoparticles (HGNPs) are core-shelled gold nanostructures with a hollow interior (Melancon et al. 2011). Attached to a homing ligand, polyethylene glycol (PEG)-coated HGNPs can be administered systemically for active targeting of tumor cells (Braun et al. 2009; Melancon et al. 2008). The strong SPR absorption at near-infrared (NIR) wavelengths induces potent photothermal effects for photoacoustic tomography (Lu et al. 2010a, 2011) and photothermal ablation applications (Melancon et al. 2008). HGNPs have also been shown to mediate light-triggered release of chemotherapeutic agents (You et al. 2010) and therapeutic small-interfering RNAs (siRNA) (Braun et al. 2009; Lu et al. 2010b).

Several mechanisms are used to trigger the release of drugs from Au-based photothermal-converting nanoparticles. In doxorubicin-loaded HGNPs, the release of doxorubicin is activated by NIR light through thermal desorption of doxorubicin from the $\mathrm{Au}$ surface (You et al. 2010). Yavuz et al. (2009) took advantage of the caging effect of thermally responsive polymer-coated Au nanocages to control drug release. NIR light-triggered release of DNA is thought to result from the breakage of $\mathrm{Au}-\mathrm{S}$ bonds by a thermal energy transfer mechanism due to localized absorption of laser energy by HGNPs to generate thermal or electron heating (Chen et al. 2006; Jain et al. 2006).

In this work, we developed a NIR light-triggered drug release system that could release platinum $[\mathrm{Pt}(\mathrm{II})]$-based chemotherapeutic agents through the same mechanism. Cisplatin is one of the most effective and widely used anticancer drugs. Several research groups have reported the formation of $\mathrm{Pt}$ complexes that contain $\mathrm{Pt}-\mathrm{N} / \mathrm{Pt}-\mathrm{Cl}$ or $\mathrm{Pt}-\mathrm{N} /$ $\mathrm{Pt}-\mathrm{O}$ coordination bonds with two Pt-N bonds in the cis position (Cheng et al. 2009; Dhar and Lippard 2009; Nishiyama et al. 2003). We designed a tripeptide, acetyl-GluGlu-Cys- $\mathrm{NH}_{2}$ (Ac-EEC), to serve as both a Pt(II) chelating agent and a linker to HGNPs. The peptide was covalently bound to HGNPs via the cysteine residue to form a gold-thiol $(\mathrm{Au}-\mathrm{S})$ bond. $\mathrm{Pt}(\mathrm{II})$-incorporated HGNPs were prepared through complexation of cisplatin and Ac-EEC, where a coordination bond forms between the Pt(II) ion and carboxylate groups in the side chains of glutamic acid residues (Scheme 1). It is demonstrated that irradiation of folate receptor-targeted, Pt(II)-loaded HGNPs, i.e., F-Ac-EEC(Pt)HGNPs, with a nanosecond (ns) pulsed laser in the NIR window triggers a rapid release of Ac-EEC(Pt) and enhances the cell mortality. Ac-EEC(Pt) was released from Au surface through oxidation of Cys to a weak Au-binding species cysteic acid (Cya), a mechanism not previously appreciated for laser triggered drug release from Au nanoparticles.

\section{Methods}

\section{Synthesis of Ac-EEC peptide}

The Ac-EEC peptides were synthesized by standard solid-phase methodology using $N^{9}$-fluorenyl-methyloxycarbonyl chemistry (Fmoc)-amino acids with acid-labile side chain protecting groups. Solid phase syntheses were carried out on a Prelude automatic peptide synthesizer (Protein Technologies, Inc., Tucson, AZ) using commercially available Rink resin. The resin $(0.05-0.1 \mathrm{~g})$ was swollen and washed 5 times with $1.5 \mathrm{~mL}$ of dimethylformamide/dichloromethane (DMF) $/ \mathrm{CH}_{2} \mathrm{Cl}_{2}$. Fmoc groups were removed by 3 treatments with $1.5 \mathrm{~mL}$ of $20 \%$ piperidine/DMF for $5 \mathrm{~min}$ each. For coupling, 3-fold excesses of Fmoc-amino acids, diisopropylcarbodiimide, and 1-hydroxybenzotriazole were added to $3 \mathrm{~mL}$ of DMF/ $\mathrm{CH}_{2} \mathrm{Cl}_{2}$. This procedure was repeated once. After coupling 


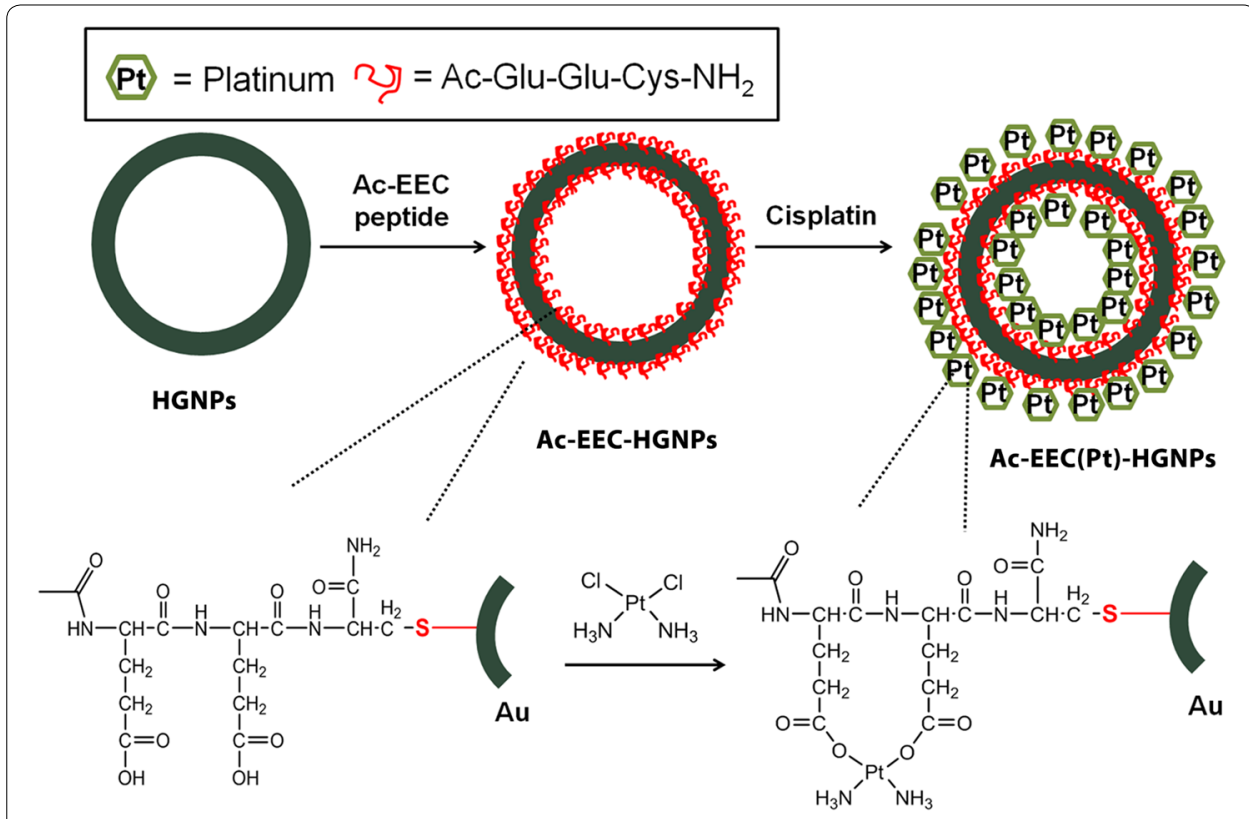

Scheme 1 Schematic illustrations of cisplatin-loaded HGNPs. The HGNPs incorporating cisplatin (Pt) are spontaneously formed via a ligand exchange reaction of Pt(II) from the chloride to the carboxylates in the Ac-EEC peptide in distilled water

and deprotection steps, the resins were washed 3 times with $3 \mathrm{~mL}$ of $\mathrm{DMF} / \mathrm{CH}_{2} \mathrm{Cl}_{2}$. On completion of the peptide chain elongation, the $\mathrm{N}$-terminal was capped by acetic anhydride, and resins were again washed 3 times with $3 \mathrm{~mL}$ of $\mathrm{CH}_{2} \mathrm{Cl}_{2}$ and were treated with a mixture of trifluoroacetic acid:triisopropylsilane: $\mathrm{H}_{2} \mathrm{O}$ (95:2.5:2.5) for $15 \mathrm{~min}$. The combined filtrates were left at room temperature for $1-2 \mathrm{~h}$, and the volumes were reduced in vacuum. Peptides were precipitated in ice-cold ethyl ether, collected by centrifugation, washed 2 times with ethyl ether. The peptides were purified by reverse-phase high-performance liquid chromatography on an Agilent 1200 system $(C-18$, Vydac, $10 \times 250 \mathrm{~mm}$, $10 \mu \mathrm{m}$; Agilent Technologies, Santa Clara, CA). Liquid chromatography-high-resolution electrospray ionization mass spectrometry (LC-MS) acquired in the positive ion mode was used to identify the peptides.

\section{Synthesis and characterization of PEG-Ac-EEC(Pt)-HGNPs and F-Ac-EEC(Pt)-HGNPs}

HGNPs were synthesized according to our previously reported procedure (Melancon et al. 2008). Briefly, cobalt nanoparticles were first synthesized by a chemical reduction method in the presence of sodium borohydride. The resulting cobalt nanoparticles served as a sacrificial template for the reduction of chloroauric acid and subsequent deposition of metallic gold on their surfaces. The obtained HGNPs nanoparticles were stabilized with sulfhydryl methoxy polyethylene glycol (MeO-PEG-SH, $5 \mathrm{kDa}$ ). Ac-EEC peptide $(4 \mathrm{mg} / \mathrm{mL}, 125 \mu \mathrm{L})$ was added to PEG-coated HGNPs $(1 \mathrm{mg})$ in $1 \mathrm{~mL}$ of water overnight at room temperature. The mixture was centrifuged and washed with deionized water three times to remove free peptide; the resulting Ac-EEC-HGNPs were mixed with $1 \mathrm{~mL}$ of aqueous cisplatin $(2.5 \mathrm{mg} / \mathrm{mL})$ and the mixture subjected to shaking at $60{ }^{\circ} \mathrm{C}$ in a water-bath for $2 \mathrm{~h}$. The solution was further shaken for $72 \mathrm{~h}$ at room temperature and 
then centrifuged at $7000 \mathrm{rpm}$ for $15 \mathrm{~min}$. The product, Ac-EEC(Pt)-HGNPs, was washed 3 times with deionized water and collected for further characterization.

Folic acid was introduced to the gold surface of Ac-EEC-HGNPs through a thioctic acid-terminated PEG linker (F-PEG-TA) according to previously reported procedures (Lu et al. 2010a). Briefly, Ac-EEC-HGNPs (1 mg) was mixed with both PEG thiol (PEG-SH; $200 \mu \mathrm{g})$ and F-PEG-TA $(50 \mu \mathrm{g})$ in $1 \mathrm{~mL}$ water at room temperature overnight to afford the folic acid-conjugated product F-Ac-EEC-HGNPs. Similarly, the non-targeting counterpart PEG-Ac-EEC-HGNPs was synthesized by mixing $250 \mu \mathrm{g}$ of PEG-SH with Ac-EEC-HGNPs without F-PEG-TA. The resulting conjugates, F-Ac-EEC-HGNPs and PEG-Ac-EEC-HGNPs, were finally complexed with cisplatin to yield F-Ac-EEC(Pt)-HGNPs and PEG-Ac-EEC(Pt)-HGNPs by following the same procedures used for the synthesis of Ac-EEC(Pt)-HGNPs.

Drug payload (Pt to Au ratio) was calculated by determining the amounts of $\mathrm{Au}$ and Pt following dissolution of the samples in aqua regia (Cheng et al. 2009). Au and $\mathrm{Pt}$ contents were quantified by inductively coupled plasma mass spectroscopy (ICP-MS; Galbraith Laboratories, Inc., Knoxville, TN) on a Varian 810 model (Varian, Inc., Walnut Creek, CA). $\mathrm{HNO}_{3}(2 \% \mathrm{v} / \mathrm{v})$ was recorded as a blank solution prior to analysis of each individual sample to monitor memory effects.

High-resolution transmission electron microscopy (HRTEM) micrographs were obtained using JEOL 2100 field emission TEM gun operating at $200 \mathrm{kV}$ (JEOL, Tokyo, Japan). TEM specimens were made by evaporating one drop of sample solution onto ultrathin carbon type-A 200 mesh copper grids (Ted Pella Inc., Redding, CA). Measurements of dynamic light scattering (DLS) were conducted with a Zetaplus (Brookhaven, Inc, Holtsville, NY) in low volume disposable cuvettes and the mean of at least three measurements was taken. The energy-dispersive X-ray spectroscopy (EDS) mapping of gold and platinum from HRTEM was obtained by using a Gatan Imaging Filter (Gatan, Inc., Pleasanton, CA). X-ray photoelectron spectroscopy (XPS) measurements were collected by using a PHI Quantera SXM XPS/ESCA system (ULVAC-PHI, Inc., Kanagawa, Japan). A monochromatic Al X-ray source at $100 \mathrm{~W}$ was used with an analytical spot size of $0.15 \mathrm{~mm} \times 1.4 \mathrm{~mm}$ and a $45^{\circ}$ takeoff angle, with pass energy of $26.00 \mathrm{eV}$. Unless noted, samples were referenced against an internal $\mathrm{Au} 4 \mathrm{f}_{7 / 2}$ line at $84.0 \mathrm{eV}$ or Pt $4 \mathrm{f}_{7 / 2}$ line at $71.2 \mathrm{eV}$. Powder XPS photoelectron lines were referenced against the $\mathrm{C} 1 \mathrm{~s}$ signal at $284.50 \mathrm{eV}$.

\section{Release of Pt from PEG-Ac-EEC(Pt)-HGNPs}

PEG-Ac-EEC(Pt)-HGNPs $(0.4 \mathrm{mg} / \mathrm{mL})$ were dispersed in $2.0 \mathrm{~mL}$ deionized water or saline solution in a $5-\mathrm{mL}$ test tube at room temperature. An NIR laser with a peak centered at $808 \mathrm{~nm}$ was generated from a master oscillator power amplifier $\mathrm{Ti}^{3+}$ :sapphire tunable laser (LT-2211A, LOTIS TII, Minsk, Republic of Belarus) pumped by a pulsed Q-switched Nd:YAG laser system (LS-2137/2, LOTIS TII) with the following parameters: wavelength, $532 \mathrm{~nm}$; pulse duration, $15 \mathrm{~ns}$; pulse energy, $400 \mathrm{~mJ}$; laser beam diameter, $7.0 \mathrm{~mm}$. The laser beam was expanded through a concave lens to a spot size of about $1 \mathrm{~cm}$ in diameter. A PM50-10 analog optical power meter with an S212A sensor (Thorlabs, Newton, NJ) was used to measure the power 
of the laser beam at the sample position. At predetermined time intervals, the samples were irradiated with the NIR laser. The HGNPs solutions were subjected to centrifugation $(14,000 \mathrm{rpm}, 20 \mathrm{~min})$ and the supernatants withdrawn for analysis of $\mathrm{Pt}$ before and after NIR laser irradiation by ICP-MS.

In a separate experiment, PEG-Ac-EEC(Pt)-HGNPs were dispersed in $2.0 \mathrm{~mL}$ deionized water or saline solution without laser irradiation at room temperature. The Pt concentration was analyzed at predetermined time intervals.

\section{Identification of Pt-containing compounds released from PEG-AC-EEC(Pt)-HGNPs}

To identify the Pt derivatives released after laser irradiation, Ac-Glu-Glu-Cysteic acid- $\mathrm{NH}_{2}$ (Ac-EECya) was synthesized using Rink amide resin and Fmoc chemistry as described above. The complex of cisplatin and Ac-EECya peptide, Ac-EECya(Pt), was synthesized by mixing cisplatin and Ac-EECya in water at $37{ }^{\circ} \mathrm{C}$ for $72 \mathrm{~h}$. LC-MS analysis was applied to aliquots collected from both PEG-Ac-EEC(Pt)-HGNPs and F-AcEEC(Pt)-HGNPs after laser treatment, and the resulting chromatograms and mass spectra were compared with that of the authentic Ac-EECya(Pt) to confirm the structure of the released Pt-containing species.

\section{Cytotoxicity}

Folate receptor-expressing KB cells were seeded in 96 -well plates $\left(2.0 \times 10^{4}\right.$ cells/well $)$ and incubated for $24 \mathrm{~h}$ to allow the cells to attach. The cells were exposed to free cisplatin, PEG-Ac-EEC(Pt)-HGNPs, or F-Ac-EEC(Pt)-HGNPs with various Pt concentrations. After $4 \mathrm{~h}$, the cells were irradiated with 15-ns pulsed NIR laser at an output power of $50 \mathrm{~mW} / \mathrm{cm}^{2}$ ( $1 \mathrm{~min} /$ treatment, 3 treatments in $2 \mathrm{~h}$ ). The cells were then incubated at $37^{\circ} \mathrm{C}$ for an additional 24 or $48 \mathrm{~h}$ without washing steps. Cell survival efficiency was measured by the (3-[4,5-dimethylthiazol-2-yl]-2,5 diphenyl tetrazolium bromide), or MTT, assay (Sigma, St Louis, MO) according to manufacturer-suggested procedures. The data are reported as the means of triplicate measurements.

In a separate experiment, KB cells were seeded onto a 96-well plate at a density of 20,000 cells/well $24 \mathrm{~h}$ before the experiment. Cells were washed 3 times with Dulbecco modified essential medium (DMEM) without phenol red. Cells were incubated with $100 \mu \mathrm{L}$ of DMEM containing F-Ac-EEC-HGNPs $(17 \mu \mathrm{M}$ of Au) or F-Ac-EEC(Pt)HGNPs $\left(17 \mu \mathrm{M}\right.$ of $\mathrm{Au}$ and $5 \mu \mathrm{M}$ of Pt) at $37{ }^{\circ} \mathrm{C}$ for $4 \mathrm{~h}$. The cells were then washed 3 times with phosphate-buffered saline solution to remove unbound nanoparticles. After cells were resupplied with DMEM containing $10 \%$ fetal bovine serum, they were irradiated with 15-ns pulsed NIR laser light centered at $808 \mathrm{~nm}$ at an output power of $50 \mathrm{~mW} /$ $\mathrm{cm}^{2}(1 \mathrm{~min} /$ treatment, 3 treatments in $2 \mathrm{~h})$ and then incubated at $37{ }^{\circ} \mathrm{C}$. Twenty after laser treatment, cells were washed 3 times with Hanks balanced salt solution and stained with calcein AM (Life Technologies, Grand Island, NY) for visualization of live cells. Untreated cells were used as controls. Cells were examined on a Zeiss Axio Observer. Z1 fluorescence microscope (Carl Zeiss MicroImaging GmbH, Göttingen, Germany) equipped with a filter set specific for excitation/emission wavelengths at $494 / 517 \mathrm{~nm}$ for calcein staining. 

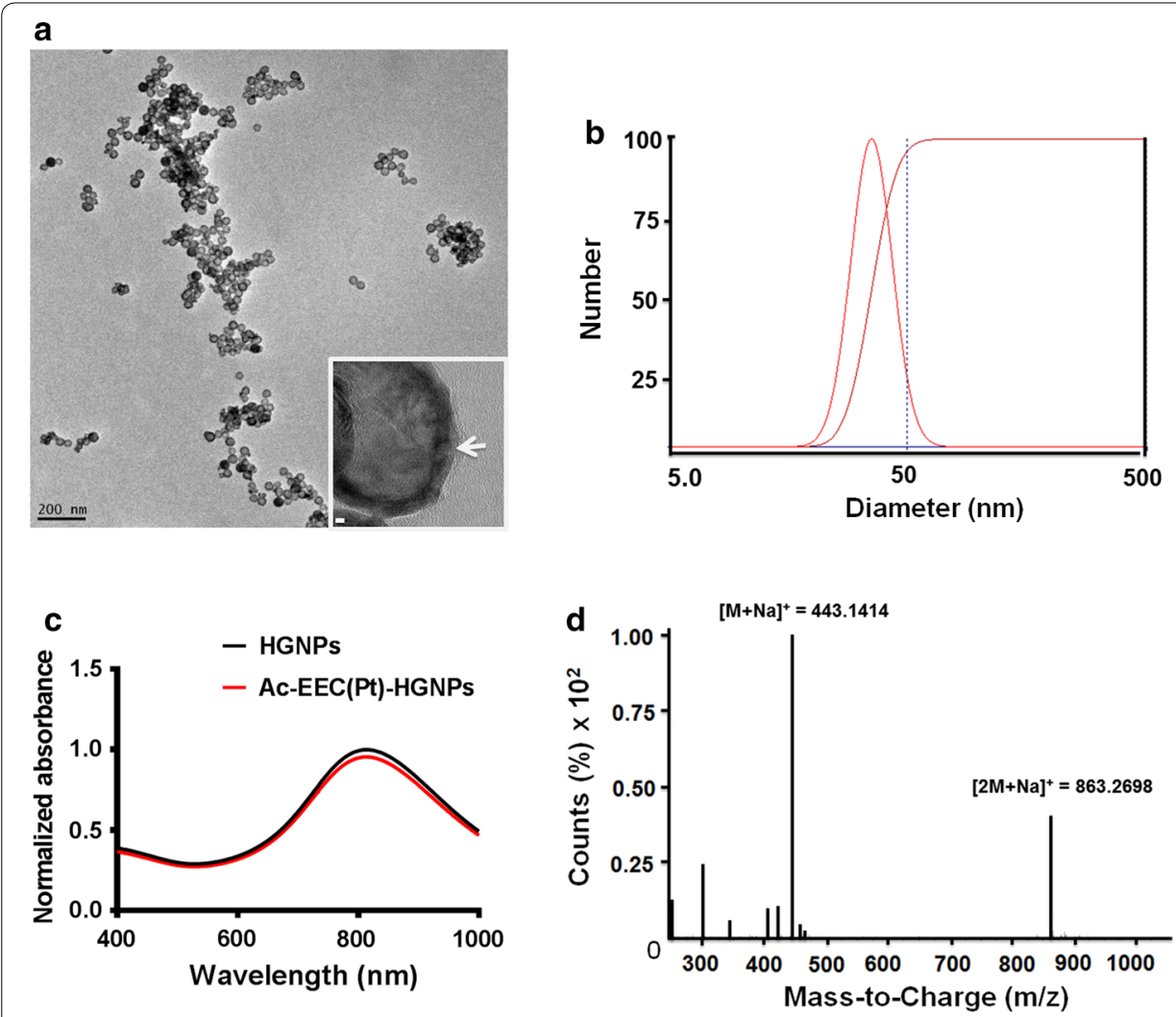

Fig. 1 Characterization of HGNPs loaded with Pt. a Representative HRTEM micrograph of Ac-EEC(Pt)-HGNPs. Arrow, nanopore on Au shell. Scale bar in inset, $2 \mathrm{~nm}$. b Representative DLS curve showing size distribution of Ac-EEC(Pt)-HGNPs. c UV-Vis-NIR spectrum of HGNPs and Ac-EEC(Pt)-HGNPs. $\mathbf{d}$ Mass spectrum of Ac-EEC peptide with positive ion mode

\section{Results and discussion}

\section{Characterization of PEG-Ac-EEC(Pt)-HGNPs}

In PEG-Ac-EEC(Pt)-HGNPs, Pt loading was achieved through a linker peptide, AcEEC, which contained 2 Glu units for Pt complexation and a Cys residue for conjugation to HGNPs. HRTEM illustrates that the typical Ac-EEC(Pt)-HGNPs had a diameter of $\sim 43.9 \pm 1.2 \mathrm{~nm}(\mathrm{n}=200)$, with a hollow core and a shell of $\sim 3-4 \mathrm{~nm}$ in thickness, and was porous on the surface (Fig. 1a). The size distribution of PEG-coated HGNPs was determined by dynamic light scattering; the hydrodynamic diameters of PEG-coated HGNPs varied from 20 to $65 \mathrm{~nm}$ peaking at $\sim 45 \mathrm{~nm}$ (Fig. 1b). The extinction spectra showed the plasmon resonance peak at $808 \mathrm{~nm}$ for both plain HGNPs and drug-loaded Ac-EEC(Pt)-HGNPs (Fig. 1c). The LC-MS spectrum shows that the synthetic Ac-EEC tripeptide had an exact mass of 420.1315 , giving rise to mass-to-charge $(\mathrm{m} / \mathrm{z})$ values of 443.1414 for $[\mathrm{M}+\mathrm{Na}]^{+}$and 863.2698 for $[2 \mathrm{M}+\mathrm{Na}]^{+}$(Fig. 1d). The presence of pores on the Au shell allowed Ac-EEC peptide and Pt(II) ions to diffuse into the interior of the HGNPs, as was the case for doxorubicin (You et al. 2010). The notion that Pt(II) bound to both the inner and the outer surfaces of the shell layer was supported by the observed distribution of Pt throughout HGNPs, as shown on EDS analysis (Fig. 2a). The presence of both Pt(II) and Au in HGNPs was confirmed by XPS, which showed the presence of 

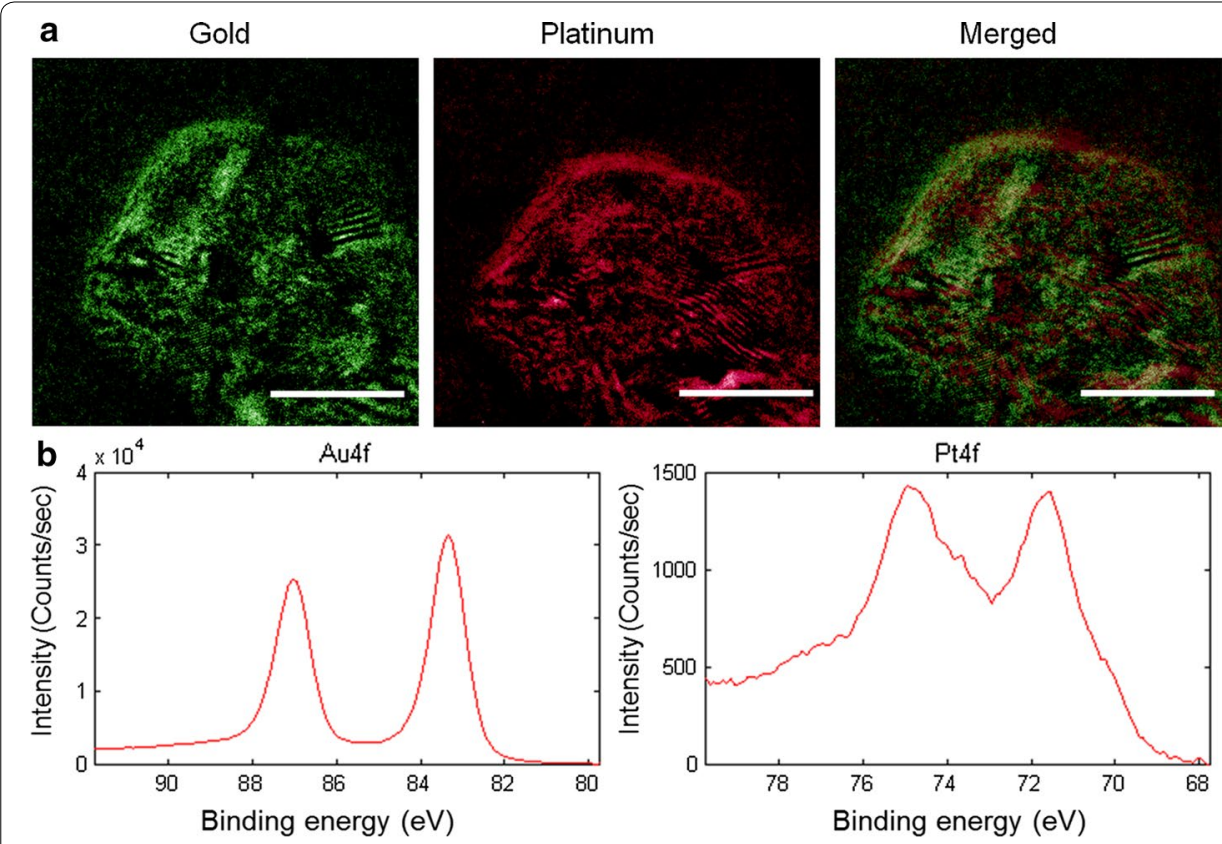

Fig. 2 Characterization of HGNPs loaded with Pt. a HRTEM/EDS micrographs mapping distribution of Au and Pt elements in Ac-EEC(Pt)-HGNPs. b XPS analysis of Au and Pt in Ac-EEC(Pt)-HGNPs
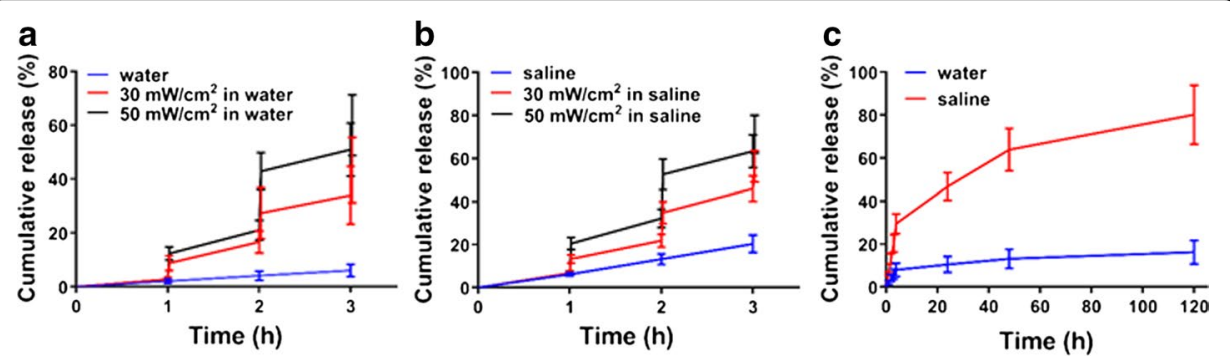

Fig. 3 Short pulsed laser induced rapid release of Pt. a Pt(II) release from Ac-EEC(Pt)-HGNPs in deionized water with or without NIR laser treatment at 30 or $50 \mathrm{~mW} / \mathrm{cm}^{2}$. b Pt(II) release from Ac-EEC(Pt)-HGNPs in saline solution with or without NIR laser treatment at 30 or $50 \mathrm{~mW} / \mathrm{cm}^{2}$. In the laser treatment groups, Nd:YAG pulsed laser (at $808 \mathrm{~nm}$ ) was applied at the time of the arrows indicated for 1 min duration. c Sustained release of Pt in water or saline solution without laser irradiation. Data are presented as means and standard deviation of 3 experiments

both Pt4f and Au4f peaks (Fig. 2b). Because Pt(II) was bound to both inner and outer surfaces of HGNPs, the resulting HGNPs-Pt had a relatively high Pt(II) payload. PEGAc-EEC(Pt)-HGNPs contained $21.9 \%$ by weight of Pt(II) as revealed by ICP-MS analysis.

\section{NIR laser-triggered $\mathrm{Pt}(\mathrm{II})$ release}

We next investigated Pt(II) release from PEG-Ac-EEC(Pt)-HGNPs with or without laser irradiation in the presence or absence of chloride ions. Figure 3 illustrates that Pt-containing compounds were released from PEG-Ac-EEC(Pt)-HGNPs upon NIR 
laser irradiation. In deionized water, the cumulative release, defined as the percentage of released Pt, increased from 2.8 to $8.8 \%$ during the first 1-min NIR laser exposure at an output power of $30 \mathrm{~mW} / \mathrm{cm}^{2}$. Release of Pt slowed drastically when the NIR laser was switched off over the next $1 \mathrm{~h}$ of incubation. Similar results were observed when the laser treatment protocol was repeated beginning at $2 \mathrm{~h}$. The cumulative release of Pt increased from 16.7 to $27.4 \%$ during the second 1-min treatment cycle. During the third treatment cycle (beginning at $3 \mathrm{~h}$ ), the cumulative release of Pt increased from 34.0 to $43.3 \%$. These data suggested that rapid Pt(II) release from PEG-Ac-EEC(Pt)HGNPs could be triggered by pulsed NIR laser.

The amount of Pt(II) released from PEG-Ac-EEC(Pt)-HGNPs could be modulated by controlling the laser power. Thus, increasing laser power from 30 to $50 \mathrm{~mW} / \mathrm{cm}^{2}$ (but keeping the treatment duration to $1 \mathrm{~min}$ ) resulted in an increase of the cumulative $\mathrm{Pt}(\mathrm{II})$ release from 8.8 to $12.4 \%$ during the first treatment cycle, from 27.4 to $42.9 \%$ during the second treatment cycle, and from 43.3 to $60.0 \%$ during the third treatment cycle (Fig. 3a). Without laser treatment, the cumulative Pt(II) release was only $6.1 \%$ during the first $3 \mathrm{~h}$. Similar laser-triggered Pt(II) release profiles were observed when PEG-AcEEC(Pt)-HGNPs was incubated in saline solution (Fig. 3b). However, the presence of chloride ions increased the release of Pt from the $6.1 \%$ observed in water to $20.4 \%$ over the $3 \mathrm{~h}$ incubation period. In saline solution, the total amounts of $\mathrm{Pt}(\mathrm{II})$ released following 3 cycles of NIR laser treatment ( 1 min treatment for each cycle) at 30 and $50 \mathrm{~mW} /$ $\mathrm{cm}^{2}$ output powers were 56.3 and $71.3 \%$, respectively.

To further evaluate the effects of ion exchange on the $\mathrm{Pt}(\mathrm{II})$ release profiles, the release of Pt from PEG-Ac-EEC(Pt)-HGNPs without NIR laser exposure was recorded for $120 \mathrm{~h}$. About 29.6\% of Pt was released from PEG-Ac-EEC(Pt)-HGNPs within the first $4 \mathrm{~h}$ of incubation in saline solution. This was followed by a slower release rate, with another approximately $50 \%$ of the $\mathrm{Pt}(\mathrm{II})$ released over the next 5 days, resulting in a cumulative $\mathrm{Pt}(\mathrm{II})$ release of $80.2 \%$ after $120 \mathrm{~h}$ (Fig. 3c). In contrast, the total amount of $\mathrm{Pt}(\mathrm{II})$ released in deionized water was only $16.3 \%$ over the same time period. In PEG-Ac-EEC(Pt)HGNPs, a coordination bond formed between the Pt(II) ion and carboxylic groups in the side chains of glutamic acid residues. This design was also used in Pt-containing polymeric micelles that were prepared through the complexation between cisplatin and PEG-poly(glutamic acid) block copolymers (Nishiyama et al. 2003). The Pt-containing micelles showed slow dissociation into unimers, accompanied with a sustained cisplatin release in physiological saline solution due to the inverse ligand exchange reaction of $\mathrm{Pt}$ (II) from the carboxylates in the copolymer to the chloride ions. However, very little Pt was released from the micelles in distilled water (Nishiyama et al. 2003). Our findings agree with the results from Pt-containing polymeric micelles that showed sustained $\mathrm{Pt}(\mathrm{II})$ release profiles in the presence of chloride ions.

\section{Modes of $\mathrm{Pt}(\mathrm{II})$ release upon laser irradiation}

The results just described demonstrate two Pt(II) release mechanisms from PEG-AcEEC(Pt)-HGNPs. First, Pt could be released from PEG-Ac-EEC(Pt)-HGNPs without NIR laser treatment in the presence of chloride ions or other anionic species that compete with carboxylates for Pt complexation, resulting in sustained release of Pt species. 


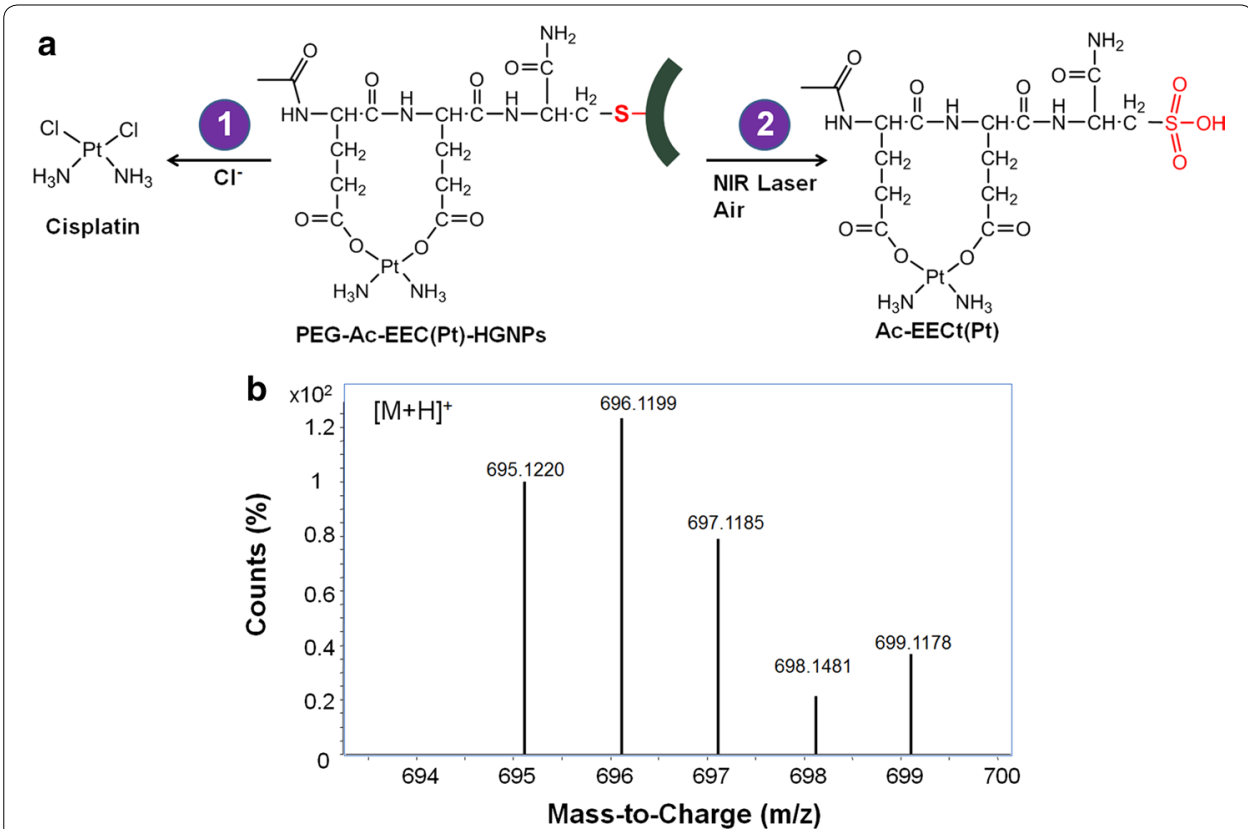

Fig. 4 Proposed modes of Pt(II) release from Ac-EEC(Pt)-HGNPs. a Schematic illustrations of Pt(II) release modes. In release mode 1, Pt(II) complexed to Ac-EEC attached to HGNPs dissociates, accompanied by sustained release of Pt via an inverse ligand exchange reaction from Glu carboxylates to the chloride ions in physiological saline solution. In release mode 2, rapid release of Pt-complexed to Ac-EEC is triggered by NIR laser irradiation through cleavage of $\mathrm{Au}-\mathrm{S}$ bonds. The compound $\mathrm{Ac}-\mathrm{EECya}(\mathrm{Pt})$ detaches from the Au surface as the Cys is oxidized to cysteic acid (Cya). b Mass spectrum of Ac-EECya-Pt(II) released from HGNPs upon NIR laser irradiation. LC-MS analysis of the released Ac-EECya-Pt was done with ESI positive ion mode. The isotopic $[\mathrm{M}+\mathrm{H}]^{+}$peaks and the corresponding abundance match those of calculated values for Ac-EECya-Pt

Second, Pt(II) release could be induced rapidly from PEG-Ac-EEC(Pt)-HGNPs upon NIR laser irradiation (Fig. 4a).

Direct $\mathrm{Au}-\mathrm{S}$ bond cleavage has been thought to underlie fast pulsed laser-triggered release of thiolated plasmid DNA for gene transfection and siRNA for RNA interference (Braun et al. 2009; Chen et al. 2006; Lu et al. 2010a; Takahashi et al. 2005; Wijaya et al. 2009). Similarly, the Ac-EEC(Pt) peptide complexes may be cleaved from HGNPs owing to a photothermal effect of HGNPs that confines thermal energy to the immediate vicinity of HGNPs with short pulsed laser. Under such conditions, Au-S bonds could be cleaved with either thermal energy transfer (Chen et al. 2006) or confined hot electrons (Jain et al. 2006). In the latter case, the hot electrons from the Au plasmons destabilizes $\mathrm{Au}-\mathrm{S}$ bonds before the electrons had thermalized with the lattice of the material (Jain et al. 2006). Alternatively, Au-S bonds may break because of oxidative desorption in which sulfhydryl in Cys is converted to sulfonate in Cya that has substantially weaker bonding with Au than the corresponding thiolate (Huang et al. 1994) (Fig. 4a). To account for this possibility, aliquots from PEG-Ac-EEC(Pt)-HGNPs after exposure to ns-pulsed laser in air was subjected to LC-MS analysis. LC-MS spectra showed prominent peaks with $\mathrm{m} / \mathrm{z}$ values ranging from 695.1220 to 699.1178 (Fig. 4b). These isotopic peaks matched calculated values for the $[\mathrm{M}+\mathrm{H}]^{+}$peaks of Ac-EECya(Pt), confirming that oxidization of the $\mathrm{Au}-\mathrm{S}$ bond to $\mathrm{Au}-\mathrm{S}(=\mathrm{O})(=\mathrm{O})$ could be an important mechanism underlying $\mathrm{Pt}(\mathrm{II})$ release from HGNPs with ns-pulsed laser. 


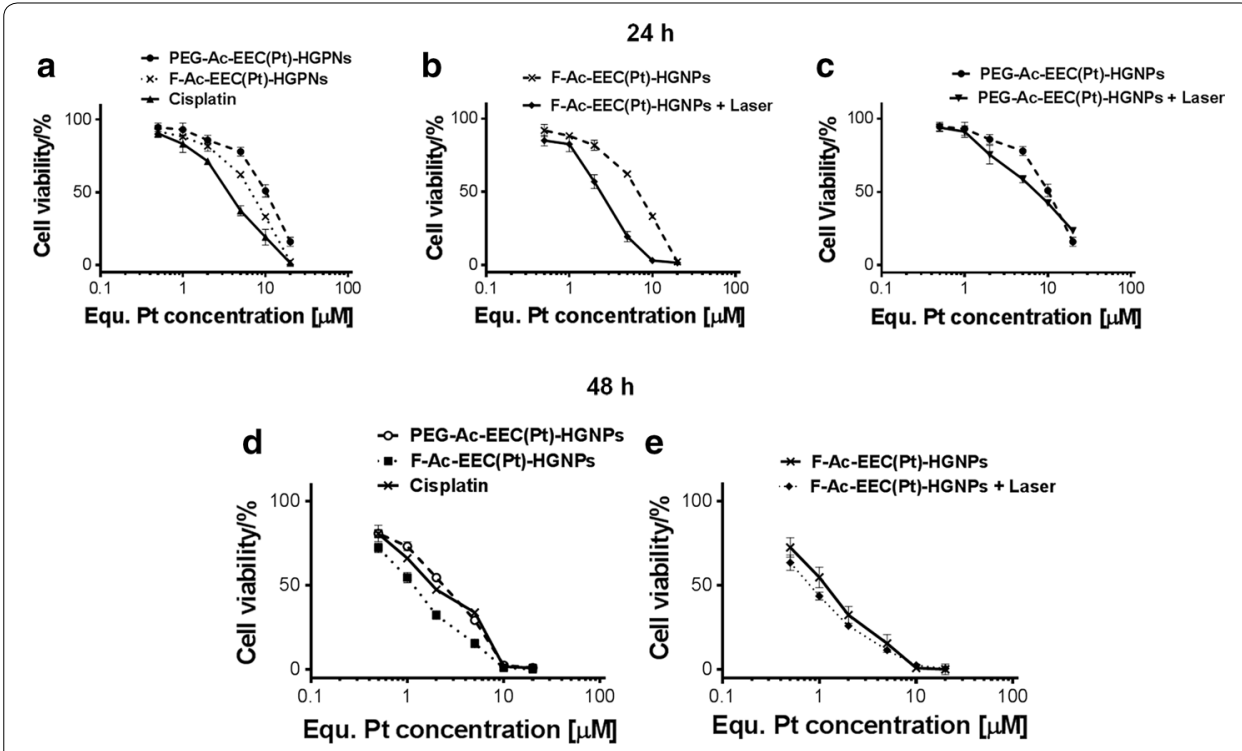

Fig. 5 Treatment with F-Ac-EEC(Pt)-HGNPs plus NIR laser exposure has greater cell mortality than F-Ac-EEC(Pt)-HGNPs alone after $24 \mathrm{~h}$ incubation. Viability of folate receptor-overexpressing KB cells was determined by MTT assay after treatments shown after $24 \mathrm{~h}(\mathbf{a}-\mathbf{c})$ and $48 \mathrm{~h}(\mathbf{d}, \mathbf{e})$ of incubation

\section{In vitro anticancer effects}

We used folate receptor-overexpressing $\mathrm{KB}$ cells to investigate cellular targeting of folic acid-conjugated Ac-EEC(Pt)-HGNPs to tumor cells (Werner et al. 2011). After $24 \mathrm{~h}$ of incubation, F-Ac-EEC(Pt)-HGNPs was more cytotoxic against KB cells than nontargeted PEG-Ac-EEC(Pt)-HGNPs. However, F-Ac-EEC(Pt)-HGNPs had lower cytotoxicity than the equivalent dose of free cisplatin. The calculated $\mathrm{IC}_{50}$ values for free cisplatin, F-Ac-EEC(Pt)-HGNPs, and PEG-Ac-EEC(Pt)-HGNPs were $3.37 \mu \mathrm{M}(95 \%$ confidence interval [CI] 2.67-4.26 $\mu \mathrm{M}), 5.68 \mu \mathrm{M}(95 \% \mathrm{CI} 4.42-7.31 \mu \mathrm{M})$, and $9.73 \mu \mathrm{M}(95 \%$ CI 7.54-12.56 $\mu \mathrm{M}$ ), respectively (Fig. 5a). Laser treatment had a greater effect on cells treated with F-Ac-EEC(Pt)-HGNPs than on cells treated with non-targeted PEG-AcEEC(Pt)-HGNPs (Fig. 5b, c). This is probably because the nanoparticles internalized into the cells via endocytosis were first sequestered in the endosomal compartments instead of the cytoplasm, which limited the transport of the Pt agent to cell nuclei to form DNA adducts. In addition, the rate of Pt(II) release from HGNPs was relatively slow without laser exposure. In contrast, free cisplatin can passively diffuse through the cell membrane into the cytoplasm and quickly accumulate in the cell nuclei (Cheng et al. 2009).

After $48 \mathrm{~h}$ of incubation, PEG-Ac-EEC(Pt)-HGNPs and F-Ac-EEC(Pt)-HGNPs exhibited $\mathrm{IC}_{50}$ values of $2.02 \mu \mathrm{M}(95 \% \mathrm{CI} 1.66-2.45 \mu \mathrm{M})$ and $1.06 \mu \mathrm{M}(95 \%$ CI $0.91-1.23 \mu \mathrm{M})$, respectively (Fig. $5 \mathrm{~d}$ ), lower than the $\mathrm{IC}_{50}$ values of the corresponding compounds after 24 $\mathrm{h}$ of incubation $(9.73 \mu \mathrm{M}$ for PEG-Ac-EEC(Pt)-HGNPs, $5.68 \mu \mathrm{M}$ for F-Ac-EEC(Pt)HGNPs). These values were similar to the $\mathrm{IC}_{50}$ value of cisplatin $(1.8 \mu \mathrm{M})$ after $48 \mathrm{~h}$ of incubation, suggesting that the benefit of targeted delivery diminished over time. Similar cytotoxicity was also observed after treatment with F-Ac-EEC(Pt)-HGNPs alone $\left(\mathrm{IC}_{50}=1.06 \mu \mathrm{M}\right)$ and F-Ac-EEC(Pt)-HGNPs plus laser $\left(\mathrm{IC}_{50}=0.75 \mu \mathrm{M}, 95 \%\right.$ CI $0.67-$ $0.85 \mu \mathrm{M})$ (Fig. 5e) after the 48-h incubation period. These data suggest that the cytotoxic 


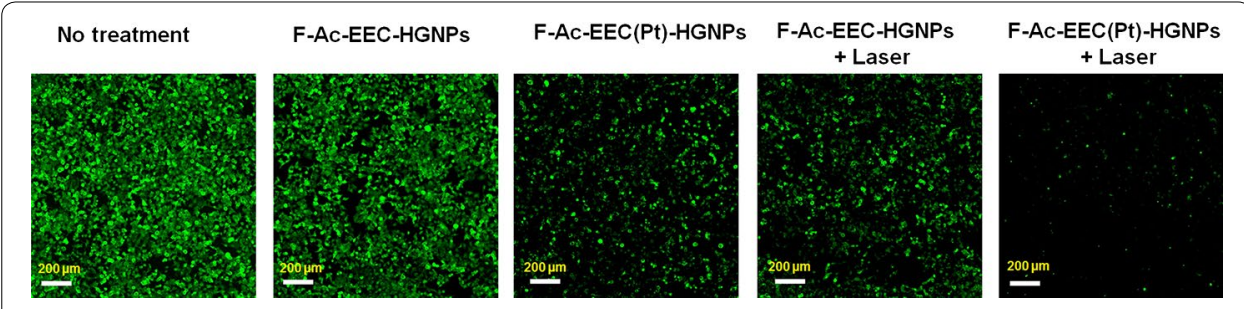

Fig. 6 Microphotographs showing enhanced cell killing with combined F-Ac-EEC-HGNPs and laser treatments. KB cells were exposed to F-Ac-EEC-HGNPs without Pt loading or F-Ac-EEC(Pt)-HGNPs with Pt loading for $4 \mathrm{~h}$. The cells were or were not irradiated with a pulsed laser at $808 \mathrm{~nm}\left(50 \mathrm{~mW} / \mathrm{cm}^{2} ; 1 \mathrm{~min}\right)$. After washing steps and an additional $20 \mathrm{~h}$ incubation, cells were stained with calcein AM. Green fluorescence represents viable cells. Bar, $200 \mu \mathrm{m}$

effect of the Pt(II) released from F-Ac-EEC(Pt) HGNPs became the predominant mechanism of increasing cell mortality after a longer incubation period.

To further evaluate the role of targeting ligand on Ac-EEC(Pt)-HGNPs in vitro, live KB cells were stained with calcein AM $20 \mathrm{~h}$ after laser treatment (Fig. 6). This experiment differed from the previous cytotoxicity study in that cells were only exposed to nanoparticles for $4 \mathrm{~h}$, then nanoparticles were removed by repeated washing steps before laser treatment was instituted. Under such conditions, both F-Ac-EEC-HGNPs and F-AcEEC(Pt)-HGNPs mediated greater effect on cell mortality against KB cells when combined with laser treatment than treatment with the corresponding nanoparticles alone (Fig. 6). Cells treated with F-Ac-EEC(Pt)-HGNPs plus laser exhibited $~ 85 \%$ decrease in fluorescence intensity compared to untreated control cells. Cells treated with F-AcEEC(Pt)-HGNPs alone or F-Ac-EEC-HGNPs (without Pt) plus laser showed moderately lower viability than untreated control cells ( 40-45\% decrease in fluorescence intensity). There was no difference in viability between cells treated with F-Ac-EEC-HGNPs alone and untreated cells, indicating the low cytotoxicity of F-Ac-EEC-HGNPs when these nanoparticles were not loaded with Pt. These results suggest that laser-triggered release of Pt(II) from F-Ac-EEC(Pt)-HGNPs increased the cell mortality of photothermolysis.

\section{Conclusions}

In this study, we synthesized and characterized Pt(II)-loaded HGNPs using a tripeptidelinker, Ac-Glu-Glu-Cys- $\mathrm{NH}_{2}$, designed to serve both as a Pt(II) chelating agent and a linker to HGNPs. The resulting Pt-HGNPs nano-carrier system displayed 2 release mechanisms. In the presence of chloride ions, the $\mathrm{Pt}(\mathrm{II})$ was released in a sustained fashion by an ion-exchange process. Upon NIR laser irradiation, oxidative desorption occurred that resulted in cleavage of $\mathrm{Au}-\mathrm{S}$ bonds and rapid release of soluble $\mathrm{Pt}(\mathrm{II}) \mathrm{com}$ pounds. F-Ac-EEC(Pt)-HGNPs combined with laser irradiation displayed greater effects on cell mortality than compared with the sole F-Ac-EEC(Pt)-HGNPs treatment over a $24 \mathrm{~h}$ incubation period.

In the clinic, photothermal ablative therapy using a Nd:YAG laser has been studied as a palliative treatment for inoperable tumors or as an alternative to more radical surgery (Paiva et al. 1998, 2002, 2005). During photothermal ablation, the tumor periphery adjacent to normal tissues can get a suboptimal thermal dose, causing reversible hyperthermic damage that can result in tumor recurrence (Paiva et al. 2005). The dual photothermal-chemotherapy approach exemplified by F-Ac-EEC(Pt)-HGNPs that release 
therapeutic agents upon laser irradiation may offer greater antitumor efficacy than photothermolysis alone mediated by plain HGNPs. Further studies on the pharmacokinetics, biodistribution, and antitumor activity of the multimodal Pt(II)-loaded HGNPs drug delivery system with NIR laser therapy are warranted.

\section{Authors' contributions}

All authors listed have made substantial, direct, and intellectual contributions to the work discussed in this publication. $C X, W L$ and $C L$ designed the article. CX, WL, MZ, WX performed the experiments and analyzed the data. WL drafted the paper. $C L$ revised the manuscript. All authors read and approved the final manuscript.

\section{Author details}

${ }^{1}$ Department of Cancer Systems Imaging, The University of Texas MD Anderson Cancer Center, Houston, USA. ${ }^{2}$ Present Address: School of Pharmacy, Fudan University, 826 Zhangheng Rd, Shanghai 201203, China. ${ }^{3}$ Present Address: Institute of Translational Medicine, School of Medicine, Zhejiang University, Hangzhou 310029, China.

\section{Acknowledgements}

We thank Kathryn Hale, Department of Scientific Publications, MD Anderson Cancer Center, for editing the manuscript.

\section{Competing interests}

The authors declare that they have no competing interests.

\section{Availability of data and materials}

Not applicable.

\section{Consent for publication}

All the authors have given consent for publication.

\section{Ethics approval and consent to participate}

Not applicable.

\section{Funding}

This work was supported in part by the National Institutes of Health (Grant R44 CA196025) and the John S. Dunn Foundation. High resolution electron microscopy facility is supported by Cancer Center Support Grant from the National Institutes of Health (P30CA016672).

\section{Publisher's Note}

Springer Nature remains neutral with regard to jurisdictional claims in published maps and institutional affiliations.

Received: 20 March 2018 Accepted: 18 June 2018

Published online: 03 August 2018

\section{References}

Bhattacharya R, Mukherjee P. Biological properties of "naked" metal nanoparticles. Adv Drug Deliv Rev. 2008;60:1289-306. Braun GB, Pallaoro A, Wu GH, Missirlis D, Zasadzinski JA, Tirrell M, Reich NO. Laser-activated gene silencing via gold nanoshell-siRNA conjugates. ACS Nano. 2009;3:2007-15.

Chen CC, et al. DNA-gold nanorod conjugates for remote control of localized gene expression by near infrared irradiation. J Am Chem Soc. 2006;128:3709-15.

Cheng Y, Samia AC, Meyers JD, Panagopoulos I, Fei B, Burda C. Highly efficient drug delivery with gold nanoparticle vectors for in vivo photodynamic therapy of cancer. J Am Chem Soc. 2008;130:10643-7.

Cheng K, Peng S, Xu C, Sun S. Porous hollow Fe(3)O(4) nanoparticles for targeted delivery and controlled release of cisplatin. J Am Chem Soc. 2009;131:10637-44.

Connor EE, Mwamuka J, Gole A, Murphy CJ, Wyatt MD. Gold nanoparticles are taken up by human cells but do not cause acute cytotoxicity. Small. 2005;1:325-7.

Dhar S, Lippard SJ. Mitaplatin, a potent fusion of cisplatin and the orphan drug dichloroacetate. Proc Natl Acad Sci USA. 2009;106:22199-204.

Ghosh PS, Kim CK, Han G, Forbes NS, Rotello VM. Efficient gene delivery vectors by tuning the surface charge density of amino acid-functionalized gold nanoparticles. ACS Nano. 2008;2:2213-8.

Gibson JD, Khanal BP, Zubarev ER. Paclitaxel-functionalized gold nanoparticles. J Am Chem Soc. 2007;129:1 1653-61.

Huang JY, Dahlgren DA, Hemminger JC. Photopatterning of self-assembled alkanethiolate monolayers on gold-a simple monolayer photoresist utilizing aqueous chemistry. Langmuir. 1994;10:626-8.

Jain PK, Qian W, El-Sayed MA. Ultrafast cooling of photoexcited electrons in gold nanoparticle-thiolated DNA conjugates involves the dissociation of the gold-thiol bond. J Am Chem Soc. 2006;128:2426-33.

Jain PK, Huang X, El-Sayed IH, El-Sayad MA. Review of some interesting surface plasmon resonance-enhanced properties of noble metal nanoparticles and their applications to biosystems. Plasmonics. 2007;2:107-18.

Kim CK, Ghosh P, Pagliuca C, Zhu ZJ, Menichetti S, Rotello VM. Entrapment of hydrophobic drugs in nanoparticle monolayers with efficient release into cancer cells. J Am Chem Soc. 2009;131:1360-1.

Lee JS, Green JJ, Love KT, Sunshine J, Langer R, Anderson DG. Gold, poly(beta-amino ester) nanoparticles for small interfering RNA delivery. Nano Lett. 2009;9:2402-6. 
Lu W, et al. Photoacoustic imaging of living mouse brain vasculature using hollow gold nanospheres. Biomaterials. 2010a;31:2617-26.

Lu W, Zhang G, Zhang R, Flores LG 2nd, Huang Q, Gelovani JG, Li C. Tumor site-specific silencing of NF-kappaB p65 by targeted hollow gold nanosphere-mediated photothermal transfection. Cancer Res. 2010b;70:3177-88.

Lu W, et al. Effects of photoacoustic imaging and photothermal ablation therapy mediated by targeted hollow gold nanospheres in an orthotopic mouse xenograft model of glioma. Cancer Res. 2011;71:6116-21.

Melancon MP, et al. In vitro and in vivo targeting of hollow gold nanoshells directed at epidermal growth factor receptor for photothermal ablation therapy. Mol Cancer Ther. 2008;7:1730-9.

Melancon MP, Zhou M, Li C. Cancer theranostics with near-infrared light-activatable multimodal nanoparticles. Acc Chem Res. 2011;44:947-56.

Nishiyama N, et al. Novel cisplatin-incorporated polymeric micelles can eradicate solid tumors in mice. Cancer Res. 2003;63:8977-83.

Paiva MB, Blackwell KE, Saxton RE, Calcaterra TC, Ward PH, Soudant J, Castro DJ. Palliative laser therapy for recurrent head and neck cancer: a phase II clinical study. Laryngoscope. 1998;108:1277-83.

Paiva MB, et al. Nd: YAG laser therapy for palliation of recurrent squamous cell carcinomas in the oral cavity. Lasers Surg Med. 2002:31:64-9.

Paiva MB, Bublik M, Castro DJ, Udewitz M, Wang MB, Kowalski LP, Sercarz J. Intratumor injections of cisplatin and laser thermal therapy for palliative treatment of recurrent cancer. Photomed Laser Surg. 2005;23:531-5.

Takahashi H, Niidome Y, Yamada S. Controlled release of plasmid DNA from gold nanorods induced by pulsed nearinfrared light. Chem Commun. 2005;17:2247-9.

Werner ME, et al. Folate-targeted polymeric nanoparticle formulation of docetaxel is an effective molecularly targeted radiosensitizer with efficacy dependent on the timing of radiotherapy. ACS Nano. 2011:5:8990-8.

Wijaya A, Schaffer SB, Pallares IG, Hamad-Schifferli K. Selective release of multiple DNA oligonucleotides from gold nanorods. ACS Nano. 2009;3:80-6.

Yavuz MS, et al. Gold nanocages covered by smart polymers for controlled release with near-infrared light. Nat Mater. 2009:8:935-9.

You J, Zhang G, Li C. Exceptionally high payload of doxorubicin in hollow gold nanospheres for near-infrared light-triggered drug release. ACS Nano. 2010;4:1033-41.

You J, et al. Pharmacokinetics, clearance, and biosafety of polyethylene glycol-coated hollow gold nanospheres. Part Fibre Toxicol. 2014;11:26

\section{Submit your manuscript to a SpringerOpen ${ }^{\odot}$ journal and benefit from:}

- Convenient online submission

- Rigorous peer review

- Open access: articles freely available online

- High visibility within the field

Retaining the copyright to your article

Submit your next manuscript at $\boldsymbol{\Delta}$ springeropen.com 